\title{
DNA-assembled bimetallic plasmonic nanosensors
}

\begin{abstract}
$\mathrm{Na} \mathrm{Li}^{1 *}$, Andreas $\mathrm{Tittl}^{2 *}$, Song Yue ${ }^{3}$, Harald Giessen ${ }^{2}$, Chen Song ${ }^{1}$, Baoquan Ding ${ }^{1}$ and Na Liu ${ }^{3}$
Plasmonic hybrid nanomaterials are highly desirable in advanced sensing applications. Different components in these materials undertake distinct roles and work collectively. One material component may act as an efficient light concentrator and optical probe, whereas another provides specific chemical or biological functionality. In this work, we present DNA-assembled bimetallic plasmonic nanostructures and demonstrate their application for the all-optical detection of hydrogen. Gold ( $\mathrm{Au}$ ) nanorods are functionalized with DNA strands, which serve both as linkers and seeding sites for the growth of palladium (Pd) nanocrystals and facilitate reliable positioning of $\mathrm{Pd}$ satellites around an Au nanorod at an ultrashort spacing in the nanometer range. Dark-field scattering spectra of single Au-DNA-Pd nanorods were recorded during controlled cycles of hydrogen gas exposure, and an unambiguous concentration-dependent optical response was observed. Our method enables, for the first time, the all-optical detection of hydrogen-induced phase-change processes in sub-5-nm Pd nanocrystals at the single-antenna level. By substituting the Pd satellites with other functional materials, our sensor platform can be extended to plasmonic sensing of a multitude of chemical and biological reagents, both in liquid and gaseous phases.
\end{abstract}

Light: Science \& Applications (2014) 3, e226; doi:10.1038/lsa.2014.107; published online 19 December 2014

Keywords: DNA self-assembly; hydrogen sensing; palladium; plasmonics

\section{INTRODUCTION}

Nanooptics, in particular plasmonics, has led to tremendous progress in optical sensors on the single-particle level. Recently, advanced wet chemical methods have enabled the controlled synthesis of metal nanoparticles with various shapes, sizes and configurations. ${ }^{1-4}$ Such metal nanoparticles possess remarkable material quality and are highly viable for industrial mass production. In particular, metal nanoparticles composed of materials such as gold $(\mathrm{Au})$ and silver $(\mathrm{Ag})$ exhibit excellent plasmonic properties and have been widely applied for sensing low-level and label-free analytes in complex media due to their simple and cost-effective sensing strategy. ${ }^{5-8}$ Traditionally, a sensing experiment is performed by tracking the resonance shift of a plasmonic nanoparticle in the presence of analytes, which alter its local environment. By combining multiple materials, biological or chemical functionality can be added to such well-established but mostly inert noble metal nanoparticle systems. ${ }^{9}$

Building on the rapid progress in the field of novel nanomaterials, various metal nanoparticles with hybrid components have been developed. For example, Au-Ag core-shell nanoparticles were used to successfully map the sulfide content in live cells through irreversible $\mathrm{Ag}_{2} \mathrm{~S}$ formation. ${ }^{10}$ Further applications of core-shell particles include the investigation of hydrogen catalytic processes in Au-Pd particles as well as the sensitive detection of carbon monoxide using a $\mathrm{Cu}-\mathrm{CuO}$ geometry. ${ }^{11,12}$ In the context of complex synthetic nanomachines, $\mathrm{Al}-$ Ga Janus nanoparticles were utilized for the creation of water-driven micromotors. ${ }^{13}$ Additionally, approaches such as on-wire lithography ${ }^{14}$ were used for the reliable fabrication of $\mathrm{Au}-\mathrm{Ni}-\mathrm{Pt}$ gap structures with nanometer precision.

Thus far, most hybrid metal nanomaterials have been based on direct contact between different material components, which can be detrimental when applied in plasmonic sensing because components with poor plasmonic properties can lead to increased damping in the hybrid system, yielding low sensitivity. To overcome this issue, DNA-based nanotechnology has been utilized for the fabrication of various metal nanoclusters and hybrid metal nanostructures. ${ }^{15-20}$

In this work, we demonstrate the chemical synthesis of DNAassembled bimetallic plasmonic nanoparticles, in which DNA serves as both the construction material and a natural spacer between two metal components, namely, Au nanorods and Pd satellite nanocrystals (Figure 1a). Furthermore, we demonstrate that such bimetallic nanoparticles can be used for the all-optical plasmonic detection of hydrogen in real time at room temperature. Here, the Pd nanocrystals work as a functional medium, which can absorb and release hydrogen. Previous experimental studies using single nanodevices have focused on catalytic reactions on extended Pd films ${ }^{21}$ or in Pd nanodisks with diameters down to $30 \mathrm{~nm} .^{22,23}$ By contrast, our nanosensor devices achieve, for the first time, the all-optical detection of hydrogeninduced phase changes in extremely small (sub-5-nm) Pd nanocrystals on the single-antenna level.

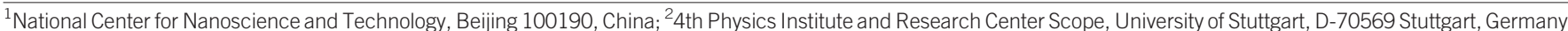
and ${ }^{3}$ Max Planck Institute for Intelligent Systems, D-70569 Stuttgart, Germany

*These authors contributed equally

Correspondence: Professor BQ Ding, National Center for Nanoscience and Technology, No. 11 ZhongGuanCun BeiYiTiao, Beijing 100190, China

E-mail: dingbq@nanoctr.cn

Or Dr N Liu, Max Planck Institute for Intelligent Systems, Heisenbergstrasse 3, D-70569 Stuttgart, Germany

E-mail: laura.liu@is.mpg.de

Received 17 June 2014; revised 20 September 2014; accepted 20 September 2014 


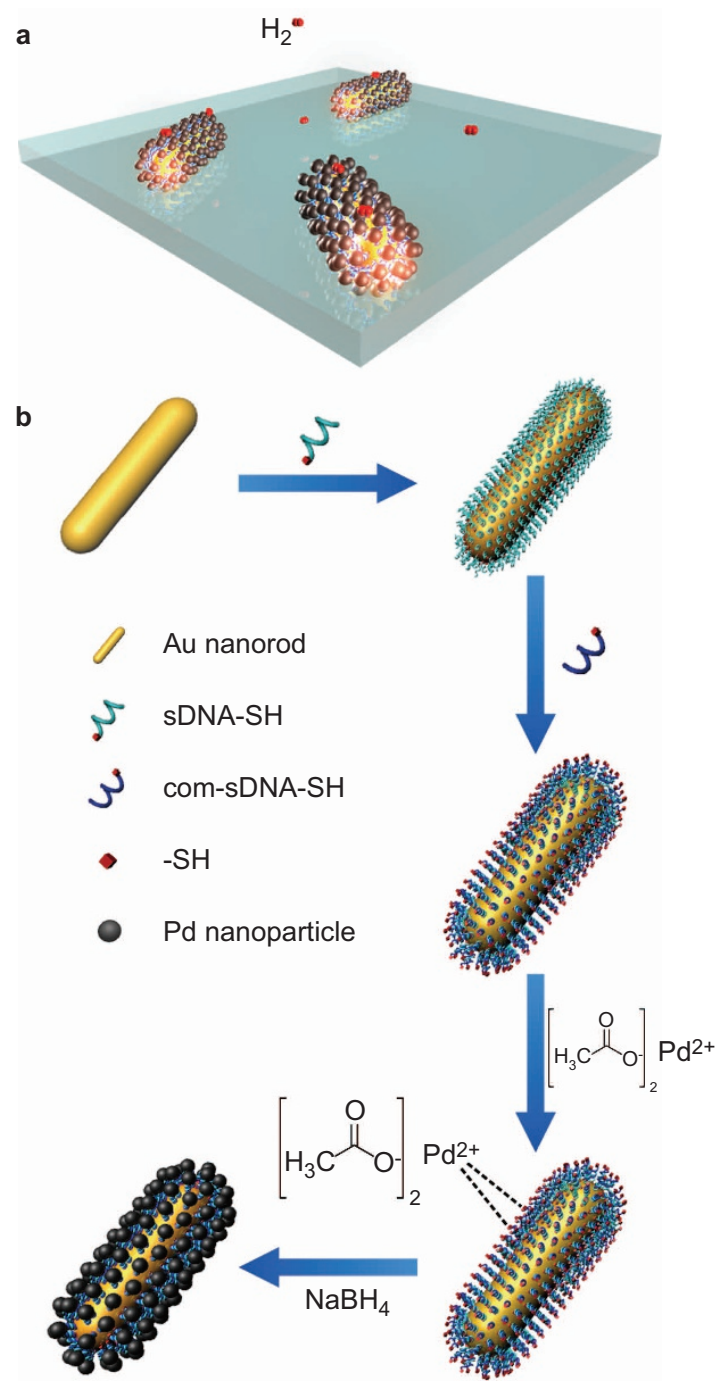

Figure 1 (a) Schematic of the DNA-assembled bimetallic nanosensor, which is composed of palladium ( $\mathrm{Pd}$ ) nanoparticles that are connected to a gold $(\mathrm{Au})$ nanorod via DNA linkers. (b) Synthesis details for the self-assembled device.

The catalytic reaction under investigation involves the chemisorption of hydrogen on the Pd surface, its near barrierless dissociation and diffusion of atomic hydrogen into Pd to form palladium hydride. ${ }^{24,25}$ The Au nanorod concentrates strong electromagnetic fields near its surface, where the chemical reactions occur. Simultaneously, the Au nanorod acts as an optical probe that transforms the localized information of hydrogen absorption and desorption in Pd to the far field.

\section{MATERIALS AND METHODS}

The preparation of our nanooptical sensor particles began with the synthesis of Au seeds, following a silver-assisted seed-growth or binary surfactant-assisted seed-growth procedure, depending on the sizes of the Au nanorods. ${ }^{1,4}$ DNA-assisted assembly and growth of the Pd satellite nanocrystals then proceeded, as illustrated in Figure 1b (further synthesis details are provided in the Supporting Information).

First, the S-S bond in thiolated DNA was reduced by adding $10 \mu \mathrm{L}$ of 100 -mM tris(2-carboxyethyl)phosphine to $50 \mu \mathrm{L}$ of $100-\mu \mathrm{M}$ DNA solution, and the sample was incubated for $4 \mathrm{~h}$. Excessive tris(2-carboxyethyl)phosphine was removed using a spin column. Subsequently, purified thiolated DNA sDNA-SH and its complementary strand com-sDNA-SH were obtained.

Freshly synthesized Au nanorods with desired dimensions were then added to the purified sDNA-SH in a buffer solution. The molar ratio of sDNA-SH to Au nanorods was more than $2000: 1$. After $12 \mathrm{~h}$, the mixture was centrifuged, the supernatant was discarded, and the $\mathrm{Au}-\mathrm{sDNA}-\mathrm{SH}$ nanorods were suspended in a $1 \times$ tris/borate/EDTA buffer containing $100-\mathrm{mM} \mathrm{NaCl}$.

The buffered $\mathrm{Au}-\mathrm{sDNA}-\mathrm{SH}$ nanorods were then incubated with the complementary com-sDNA-SH and were annealed in a thermocycler from $45{ }^{\circ} \mathrm{C}$ to $25{ }^{\circ} \mathrm{C}$ over $12 \mathrm{~h}$. The mixture was then centrifuged twice, the supernatant was discarded, and the pellet was suspended in ultrapure water. The concentration of these Au-DNA conjugates was estimated using ultraviolet-visible spectroscopy.

The Au-DNA nanorods were then mixed with $20 \mu \mathrm{L}$ of a freshly prepared $2.5-\mathrm{mM} \mathrm{Pd}\left(\mathrm{CH}_{3} \mathrm{COO}\right)_{2}$ solution and $20 \mu \mathrm{L}$ of $1 \%$ polyvinylpyrrolidone. The mixture was stirred for $4 \mathrm{~h}$ at room temperature and subsequently centrifuged three times at $5000 \mathrm{rpm}$ for $5 \mathrm{~min}$. Again, the supernatant was discarded and the pellet was suspended in ultrapure water. Finally, the mixture was reduced by $10 \mu \mathrm{L}$ of $5-\mathrm{mM}$ freshly prepared ice-cold $\mathrm{NaBH}_{4}$ to obtain the Au-DNA-Pd hybrid nanoparticles.

\section{RESULTS AND DISCUSSION}

To first assess the quality of our Au-DNA-Pd nanoparticles, transmission electron microscopy (TEM), scanning TEM (STEM) and elemental mapping using a Tecnai F20 electron microscopy system (FEI Corp., Hillsboro, OR, USA) were performed. The samples for TEM imaging were prepared by drop-casting $10 \mu \mathrm{L}$ of the sample solution on a carbon-coated TEM grid (additional measurement details are provided in the Supporting Information).

Figure 2 presents the TEM images of the Au-DNA-Pd nanoparticles with different dimensions, demonstrating the high structural homogeneity of our synthesis method. The three batches of Au-DNA-Pd nanoparticles (labeled 1 to 3 ) have dimensions (length $\times$ width) of $36 \mathrm{~nm} \times 12 \mathrm{~nm}, 46 \mathrm{~nm} \times 12 \mathrm{~nm}$ and $98 \mathrm{~nm} \times 43 \mathrm{~nm}$. The Pd nanocrystals are approximately $3-5 \mathrm{~nm}$ in diameter, as evident from high-resolution TEM measurements (Figure 3a). In all cases, the individual satellite Pd nanocrystals are clearly visible at high magnifications.

Successful assembly of the different metal components was demonstrated using STEM imaging and elemental mapping of the Au-DNAPd nanoparticles from batch 1 (Figure 3a). By filtering the STEM image with respect to the electron energy in the scanning beam, the elemental contributions of $\mathrm{Au}$ and $\mathrm{Pd}$ to the total signal could be quantified (Figure 3b and 3c). A composite of the color-coded results for $\mathrm{Au}$ and Pd is presented in Figure 3d, which clearly illustrates that small Pd satellite nanocrystals form a dispersive layer around the center $\mathrm{Au}$ nanorod. This finding proves the viability of our synthesis method for the precise and reliable creation of complex hybrid nanostructures.

To investigate the optical properties of our $\mathrm{Au}-\mathrm{DNA}-\mathrm{Pd}$ nanoparticles, ultraviolet-visible spectra of the three nanorod batches, before and after the Pd growth, were measured (Figure 4). For all three batches, the pronounced plasmonic absorbance peak red-shifts by approximately $50 \mathrm{~nm}$ after the growth of the Pd nanocrystals. The hybrid plasmonic nanoparticles clearly exhibit excellent wavelength tunability through control of the structural aspect ratio.

To dissect the sensing behavior of a nanoparticle ensemble and to achieve progressively smaller detection volumes, we utilize single-particle dark-field spectroscopy. In our hydrogen sensing experiments, we 

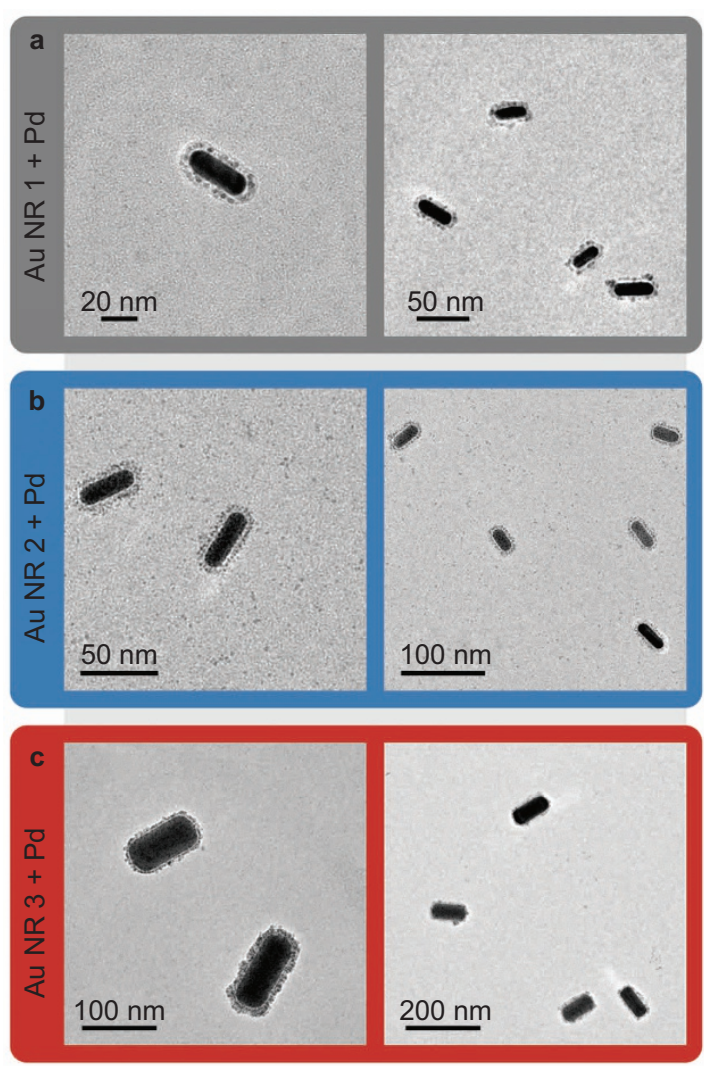

Figure 2 TEM images of the three batches of DNA-assembled nanosensors with different design dimensions. TEM, transmission electron microscopy. NR, nanorod.

recorded the scattered light from single Au-DNA-Pd nanoparticles while cycling the gas concentration in a gas cell between pure nitrogen and different $\mathrm{H}_{2}$ concentrations in $\mathrm{N}_{2}$ carrier gas (experimental details are provided in the Supporting Information). Figure 5 a presents the scattering spectrum of a single plasmonic nanosensor from batch 3 (length $\times$ width: $98 \mathrm{~nm} \times 43 \mathrm{~nm}$ ), which exhibits a pronounced peak centered at approximately $700 \mathrm{~nm}$. Compared with the ensemble measurements in aqueous solution, the single-particle resonance is slightly blue-shifted on the glass substrate.

To reliably track the resonance wavelength of the nanosensor over time, we employed a centroid detection method. ${ }^{26}$ This analysis technique computes the first moment, or center of mass, of the scattering spectrum, considering all the data points in a certain wavelength span $S_{\mathrm{c}}$. This procedure allows us to track small spectral shifts with high signal-to-noise ratios.

The resonance peak undergoes a fast and reversible red-shift when exposed to hydrogen concentrations of 10,20 and 30 vol.- $\% \mathrm{H}_{2}$ in $\mathrm{N}_{2}$ carrier gas, with response times on the order of seconds. Additionally, we observed a clear concentration-dependent response, with spectral shifts ranging from 0.2 to $0.4 \mathrm{~nm}$. When examining a second nanosensor particle on the same substrate, we again observed a clear concentration dependence of the optical response (Supplementary Fig. S1). Although the magnitude of the spectral shifts is different, the qualitative behavior is similar (Supplementary Fig. S2). This difference in magnitude is due to small changes in local material distribution and can be further reduced by optimizing the synthesis process. Most importantly, we were able to, for the first time, detect the hydrogen-induced phase-change processes in extremely small Pd particles on the order of 3-5 $\mathrm{nm}$ at the single-antenna level.

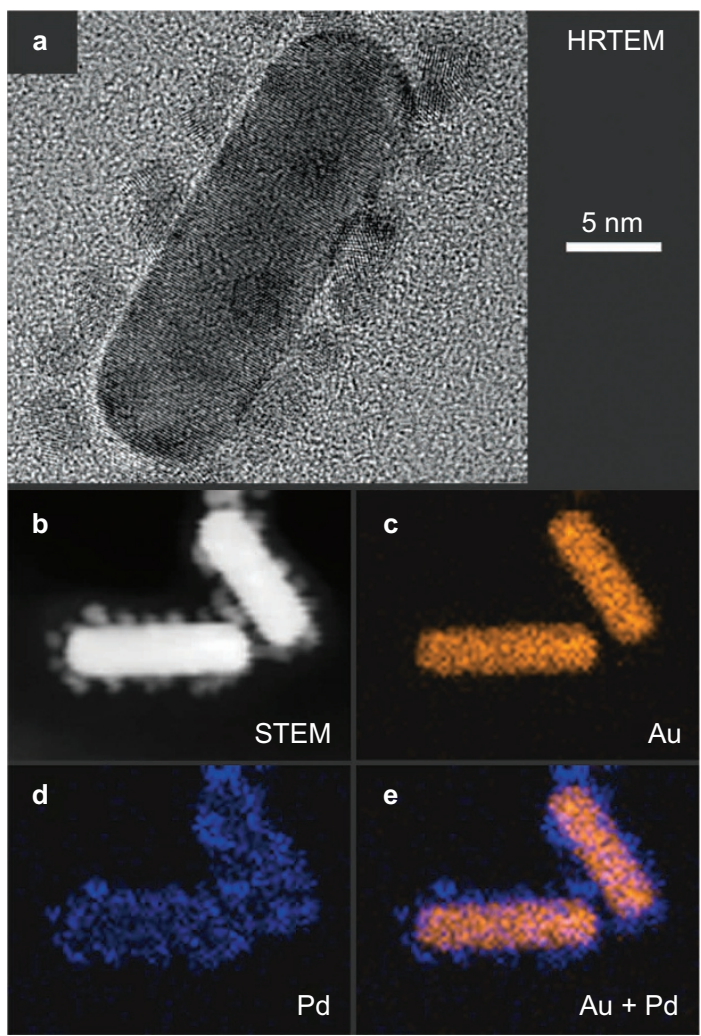

Figure 3 (a) HRTEM image of an Au-DNA-Pd nanorod from batch 1. The sub-5$\mathrm{nm}$ satellite Pd nanocrystals are clearly visible. (b) STEM image of two nanorods from batch 1. (c-e) Element-resolved (energy-filtered) STEM images of the same nanorods. HRTEM, high-resolution transmission electron microscopy; STEM, scanning transmission electron microscopy.

\section{CONCLUSIONS}

In this work, we demonstrated a novel nanooptical single-particle hydrogen sensor utilizing wet-chemical synthesis of Au nanorods with adjacent, DNA-assembled Pd nanocrystals. Our approach results in hybrid plasmonic nanoparticles with tunable particle dimensions and ultrashort $\mathrm{Au}-\mathrm{Pd}$ distances on the order of several nanometers. The

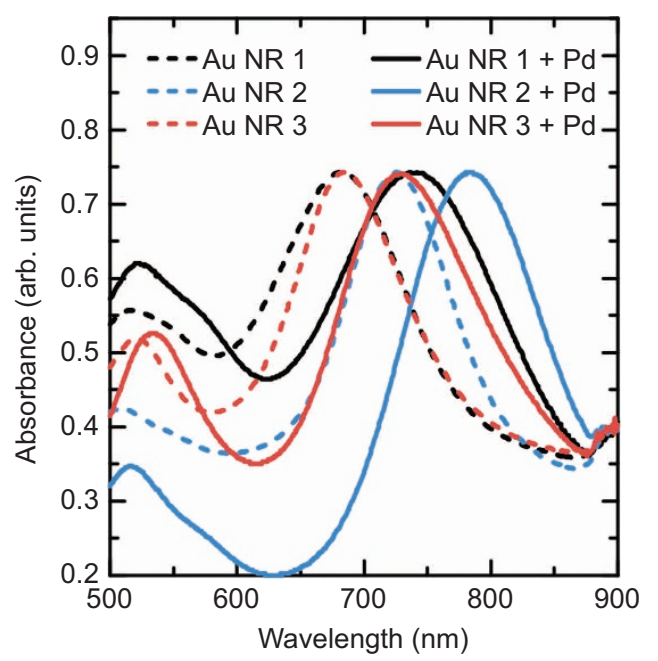

Figure 4 Ensemble absorbance spectra for three batches of Au nanorods of different design lengths, with and without DNA-assembled Pd nanocrystals (solid and dashed curves). NR, nanorod. 

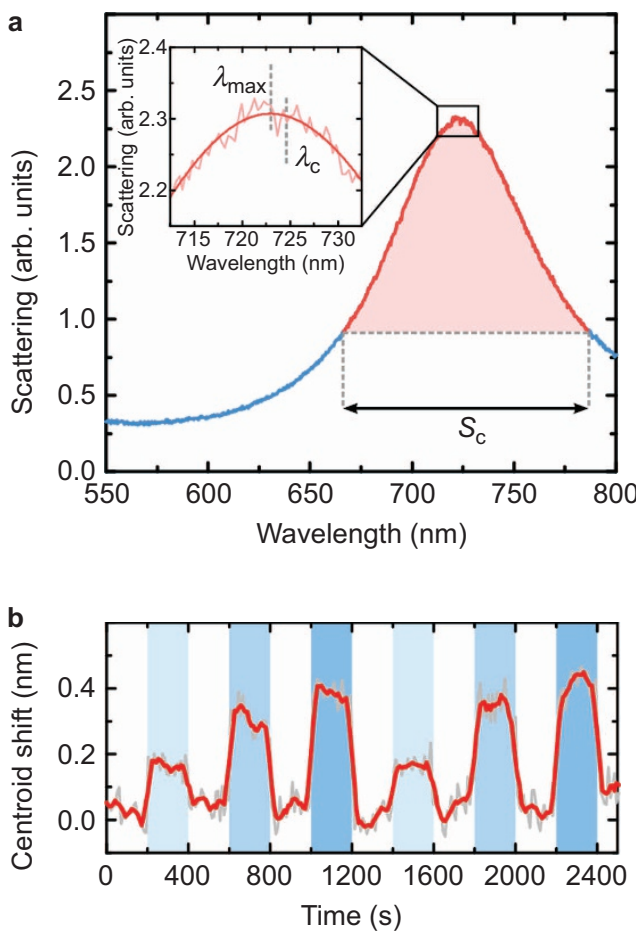

Figure 5 (a) Dark-field scattering spectrum of a single Au-DNA-Pd nanorod on a glass substrate. $S_{c}$ indicates the wavelength span included in our centroid calculation. Comparison of the wavelength of the scattering maximum $\lambda_{\max }$ and the centroid wavelength $\lambda_{\mathrm{c}}$ (inset). (b) Time-resolved sensing performance of the DNA-assembled nanosensor when exposed to cycles of 10,20 and 30 vol.- $\% \mathrm{H}_{2}$ in $\mathrm{N}_{2}$ and $\mathrm{N}_{2}$ carrier gas. The gray line represents the centroid wavelength from scattering spectra taken every $10 \mathrm{~s}$ with an integration time of $8 \mathrm{~s}$. The red line is a running average over five data points.

precise positioning of the $\mathrm{Pd}$ satellite nanospheres with respect to the $\mathrm{Au}$ nanorods was verified using energy-filtered scanning TEM measurements. Going further, the number of Pd nanocrystals linked to each Au nanorod could be controlled by the amount and ratio of thiolated DNA and complementary strands. Additionally, we demonstrated that our $\mathrm{Au}-$ DNA-Pd nanoparticles exhibit an unambiguous concentration-dependent optical response to hydrogen exposure at the single-particle level. Using appropriate satellite nanoparticle components, one could prepare hybrid plasmonic nanosensor devices with different functionalizations, enabling the reliable detection of a multitude of other gases for practical applications. In the future, plasmon-assisted detection of transformations in nanoparticles of such small sizes will open the door toward investigations of size-dependent phase transitions on nanoscales below $10 \mathrm{~nm}$.

\section{COMPETING FINANCIAL INTERESTS}

The authors declare no competing financial interests.

\section{ACKNOWLEDGEMENTS}

We thank K Hahn for help with structure imaging. AT and HG were financially supported by the Deutsche Forschungsgemeinschaft (SPP1391, FOR730, GI 269/111), the Bundesministerium für Bildung und Forschung (13N9048 and 13N10146), the ERC Advanced Grant COMPLEXPLAS, the Baden-Württemberg Stiftung (Spitzenforschung II) and the Ministerium für Wissenschaft, Forschung und Kunst Baden-Württemberg (Az: 7533-7-11.6-8). NL was supported by the Sofia
Kovalevskaja Award of the Alexander von Humboldt Foundation and Grassroots Proposal M10331 from the Max Planck Institute for Intelligent Systems. BD was supported by National Science Foundation China (21173059, 21222311, 91127021) and 100-Talent Program of the Chinese Academy of Sciences.

1 Ye X, Zheng C, Chen J, Gao Y, Murray CB. Using binary surfactant mixtures to simultaneously improve the dimensional tunability and monodispersity in the seeded growth of gold nanorods. Nano Lett 2013; 13: 765-771.

2 Burdick J, Laocharoensuk R, Wheat PM, Posner JD, Wang J. Synthetic nanomotors in microchannel networks: directional microchip motion and controlled manipulation of cargo. J Am Chem Soc 2008; 130: 8164-8165.

3 Sönnichsen C, Reinhard BM, Liphardt J, Alivisatos AP. A molecular ruler based on plasmon coupling of single gold and silver nanoparticles. Nat Biotechnol 2005; 23: 741-745.

4 Nikoobakht B, El-Sayed MA. Preparation and growth mechanism of gold nanorods (NRs) using seed-mediated growth method. Chem Mater 2003; 15: 1957-1962.

5 Mayer KM, Hafner JH. Localized surface plasmon resonance sensors. Chem Rev 2011; 111: 3828-3857.

6 Stewart ME, Anderton CR, Thompson LB, Maria J, Gray SK et al. Nanostructured plasmonic sensors. Chem Rev 2008; 108: 494-521.

7 Anker JN, Hall WP, Lyandres O, Shah NC, Zhao J et al. Biosensing with plasmonic nanosensors. Nat Mater 2008; 7: 442-453.

8 Lal S, Link S, Halas NJ. Nano-optics from sensing to waveguiding. Nat Photonics 2007; 1: 641-648.

9 A Tittl, H Giessen, N Liu. Plasmonic gas and chemical sensing. Nanophotonics 2014; 3: $157-180$.

10 Xiong B, Zhou R, Hao J, Jia Y, He Y et al. Highly sensitive sulphide mapping in live cells by kinetic spectral analysis of single $\mathrm{Au}-\mathrm{Ag}$ core-shell nanoparticles. Nat Commun 2013; 4: 1708 .

11 Tang ML, Liu N, Dionne JA, Alivisatos AP. Observations of shape-dependent hydrogen uptake trajectories from single nanocrystals. J Am Chem Soc 2011; 133: 13220-13223.

12 Ghodselahi T, Zahrabi H, Saani MH, Vesaghi MA. CO gas sensor properties of Cu@CuO core-shell nanoparticles based on localized surface plasmon resonance. J Phys Chem C2011; 115: 22126-22130.

13 Gao W, Pei A, Wang J. Water-driven micromotors. ACS Nano 2012; 6: 8432-8438.

14 Qin L, Park S, Huang L, Mirkin CA. On-wire lithography. Science 2005; 309: 113-115.

15 Deng Z, Mao C. DNA-templated fabrication of 1D parallel and 2D crossed metallic nanowire arrays. Nano Lett 2003; 3: 1545-1548.

16 Hatakeyama Y, Umetsu M, Ohara S, Kawadai F, Takami S et al. Homogenous spherical mosslike assembly of Pd nanoparticles by using DNA compaction: application of Pd-DNA hybrid materials to volume-expansion hydrogen switches. Adv Mater 2008; 20: 1122-1128.

17 Stearns LA, Chhabra R, Sharma J, Liu Y, Petuskey WT et al. Template-directed nucleation and growth of inorganic nanoparticles on DNA scaffolds. Angew Chem Int Ed 2009; 48: 8494-8496.

18 Pal S, Varghese R, Deng Z, Zhao Z, Kumar A et al. Site-specific synthesis and in situ immobilization of fluorescent silver nanoclusters on DNA nanoscaffolds by use of the Tollens reaction. Angew Chem 2011; 123: 4262-4265.

19 Schreiber R, Kempter S, Holler S, Schüller V, Schiffels D et al. DNA origami-templated growth of arbitrarily shaped metal nanoparticles. Small 2011; 7: 1795-1799.

20 Geng Y, Pearson AC, Gates EP, Uprety B, Davis RC et al. Electrically conductive goldand copper-metallized DNA origami nanostructures. Langmuir 2013; 29: 3482-3490.

21 Tittl A, Yin X, Giessen H, Tian XD, Tian ZQ et al. Plasmonic smart dust for probing local chemical reactions. Nano Lett 2013; 13: 1816-1821.

22 Liu N, Tang ML, Hentschel M, Giessen H, Alivisatos AP. Nanoantenna-enhanced gas sensing in a single tailored nanofocus. Nat Mater 2011; 10: 631-636.

23 Shegai T, Langhammer $\mathrm{C}$. Hydride formation in single palladium and magnesium nanoparticles studied by nanoplasmonic dark-field scattering spectroscopy. Adv Mater 2011; 23: 4409-4414.

24 Flanagan TB, Oates WA. The palladium-hydrogen system. Annu Rev Mater Sci 1991; 21: 269-304.

25 Poyli MA, Silkin VM, Chernov IP, Echenique PM, Muiño RD et al. Multiscale theoretical modeling of plasmonic sensing of hydrogen uptake in palladium nanodisks. J Phys Chem Lett 2012; 3: 2556-2561.

26 Dahlin AB, Tegenfeldt JO, Höök F. Improving the instrumental resolution of sensors based on localized surface plasmon resonance. Anal Chem 2006; 78: 4416-4423.

(c) (i) (2) This work is licensed under a Creative Commons AttributionNonCommercial-ShareAlike 3.0 Unported License. The images or other third party material in this article are included in the article's Creative Commons license, unless indicated otherwise in the credit line; if the material is not included under the Creative Commons license, users will need to obtain permission from the license holder to reproduce the material. To view a copy of this license, visit http://creativecommons.org/licenses/by-nc-sa/3.0/ 